\title{
Global Bifurcation from Intervals for the Monge-Ampère Equations and Its Applications
}

\author{
Wenguo Shen (1D) \\ Department of Basic Courses, Lanzhou Institute of Technology, Lanzhou 730050, China \\ Correspondence should be addressed to Wenguo Shen; shenwg369@163.com
}

Received 8 August 2017; Accepted 20 November 2017; Published 9 January 2018

Academic Editor: Vijay Gupta

Copyright (c) 2018 Wenguo Shen. This is an open access article distributed under the Creative Commons Attribution License, which permits unrestricted use, distribution, and reproduction in any medium, provided the original work is properly cited.

We shall establish the global bifurcation results from the trivial solutions axis or from infinity for the Monge-Ampère equations: $\operatorname{det}\left(D^{2} u\right)=\lambda m(x)(-u)^{N}+m(x) f_{1}\left(x,-u,-u^{\prime}, \lambda\right)+f_{2}\left(x,-u,-u^{\prime}, \lambda\right)$, in $B, u(x)=0$, on $\partial B$, where $D^{2} u=\left(\partial^{2} u / \partial x_{i} \partial x_{j}\right)$ is the Hessian matrix of $u$, where $B$ is the unit open ball of $\mathbb{R}^{N}, m \in C(\bar{B},[0,+\infty))$ is a radially symmetric weighted function and $m(r):=m(|x|) \not \equiv$ 0 on any subinterval of $[0,1], \lambda$ is a positive parameter, and the nonlinear term $f_{1}, f_{2} \in C\left(\bar{B} \times\left(\mathbb{R}^{+}\right)^{3}, \mathbb{R}^{+}\right)$, but $f_{1}$ is not necessarily differentiable at the origin and infinity with respect to $u$, where $\mathbb{R}^{+}=[0,+\infty)$. Some applications are given to the Monge-Ampère equations and we use global bifurcation techniques to prove our main results.

\section{Introduction}

The Monge-Ampère equations are a type of important fully nonlinear elliptic equations [1-3]. Existence and regularity results of the Monge-Ampère equations can be found in [4-8] and the references therein. tions:

We first consider the following real Monge-Ampère equa-

$$
\begin{aligned}
\operatorname{det}\left(D^{2} u\right) & =\lambda m(x)(-u)^{N}+F\left(x,-u,-u^{\prime}, \lambda\right), \quad \text { in } B, \\
u(x) & =0, \quad \text { on } \partial B,
\end{aligned}
$$

where $D^{2} u=\left(\partial^{2} u / \partial x_{i} \partial x_{j}\right)$ is the Hessian matrix of $u, B$ is the unit ball of $\mathbb{R}^{N}, m(x)$ is a weighted function, $\lambda$ is a positive parameter, and $F \in C\left(\bar{B} \times\left(\mathbb{R}^{+}\right)^{3}, R^{+}\right)$.

Kutev [9] and Delano [10] treated the existence of convex radial solutions of problem (1) with $m \equiv 1, F=0$ and $\lambda m(-u)^{N}+F=\lambda \exp f(|x|, u,|\nabla u|)$, respectively. Caffarelli et al. [11] have investigated problem (1) in general domains of $\mathbb{R}^{N}$.

In $[9,12]$, the authors have shown that problem (1) can reduce to the following boundary value problem:

$$
\begin{aligned}
\left(\left(u^{\prime}\right)^{N}\right)^{\prime}= & \lambda N r^{N-1} m(r)(-u)^{N} \\
& +N r^{N-1} F\left(r,-u,-u^{\prime}, \lambda\right), \quad r \in(0,1),
\end{aligned}
$$

$$
u^{\prime}(0)=u(1)=0,
$$

where $r=|x|$ with $x \in B$. By a solution of problem (2), we understand that it is a function which belongs to $C^{2}[0,1]$ and satisfies (2). It has been known that any negative solution of problem (2) is strictly convex in $(0,1)$. Wang [13] and $\mathrm{Hu}$ and Wang [12] ( $m \equiv 0 ; F=f(-u)$ ) also considered the existence of strictly convex solutions for problem (2) by using fixed index theorem. Lions [14] proved the existence of the first eigenvalue $\lambda_{1}$ of problem (1) with $\lambda m \equiv \lambda^{N}, F=0$ via constructive proof.

By global bifurcation theorem, Dai and Ma [15] and Dai [16] studied the Monge-Ampère equations (1) with $\lambda m(x)(-u)^{N}+F$ equal $\lambda^{N}\left((-u)^{N}+g(-u)\right)$ and $\lambda^{N} m(x)\left((-u)^{N}+g(-u)\right)$, respectively, where $g:[0,+\infty) \rightarrow$ $[0,+\infty)$ satisfies $\lim _{s \rightarrow 0^{+}} g(s) / s^{N}=0$.

(H0) $m(x) \in C(\bar{B})$ is radially symmetric and $m(r) \geq 0$, $m(r) \not \equiv 0$ on any subinterval of $[0,1]$, where $r=|x|$ with $x \in \bar{B}$

However, the nonlinearities of the above papers are differentiable at the origin. In 1977, Berestycki [17] established an interval bifurcation theorem for the problems involving nondifferentiable nonlinearity. The main difficulties when 
dealing with this problem lie in the bifurcation results of $[15,16]$ which cannot be applied directly to obtain our results. Recently, Ma and Dai [18] and Dai and Ma [19, 20] also considered similar problems to [17].

Motivated by the above papers, we shall consider problem (1), where $F=m(x) f_{1}+f_{2}$, with $f_{1}, f_{2} \in C\left(\bar{B} \times\left(\mathbb{R}^{+}\right)^{3}, \mathbb{R}^{+}\right)$ being radially symmetric with respect to $x$, and $\mathbb{R}^{+}=$ $[0,+\infty)$.

It is clear that the radial solutions of (1) are equivalent to the solutions of problem (2), where $m$ satisfies $(\mathrm{H} 0)$ and $f_{1}$ and $f_{2}$ satisfy the following conditions.

such that

(H1) $f_{1} \in C\left([0,1] \times\left(\mathbb{R}^{+}\right)^{3}\right)$ and there exist $a_{0}, a^{0} \in(0, \infty)$

$$
a_{0} \leq \liminf _{s \rightarrow 0^{+}} \frac{f_{1}(r, s, y, \lambda)}{s^{N}}<\limsup _{s \rightarrow 0^{+}} \frac{f_{1}(r, s, y, \lambda)}{s^{N}} \leq a^{0}
$$

uniformly for $r \in[0,1], 0<y \leq 1$, and for all $\lambda \in \mathbb{R}^{+}$.

(H2) $f_{1} \in C\left([0,1] \times\left(\mathbb{R}^{+}\right)^{3}\right)$ and there exist $a_{\infty}, a^{\infty} \in$ $(0, \infty)$ such that

$$
\begin{aligned}
a_{\infty} & \leq \liminf _{s \rightarrow+\infty} \frac{f_{1}(r, s, y, \lambda)}{s^{N}}<\limsup _{s \rightarrow+\infty} \frac{f_{1}(r, s, y, \lambda)}{s^{N}} \\
& \leq a^{\infty}
\end{aligned}
$$

uniformly for $r \in[0,1], y \geq C$ for some positive constant $C$ large enough, and for all $\lambda \in \mathbb{R}^{+}$.

(H3) $f_{2}(r, s, y, \lambda)=o\left(s^{N}+y^{N}\right)$ near $(s, y)=(0,0)$ uniformly for $r \in(0,1)$ and $\lambda$ on bounded sets.

(H4) $f_{2}(r, s, y, \lambda)=o\left(s^{N}+y^{N}\right)$ near $(s, y)=(+\infty,+\infty)$ uniformly for $r \in(0,1)$ and $\lambda$ on bounded sets.

Under assumptions (H1) and (H3), we shall establish interval bifurcation of (2) from the trivial solutions axis by Rabinowitz [21]. Moreover, by the global bifurcation from infinity of Rabinowitz [22], we shall also establish a result involving global bifurcation of (2) from infinity with conditions (H2) and (H4).

Following the above theory (see Theorems 3 and 6), we shall investigate the existence of radial solutions for the following problem:

$$
\begin{aligned}
\operatorname{det}\left(D^{2} u\right) & =\gamma h(x) F(-u), \quad \text { in } B, \\
u(x) & =0, \quad \text { on } \partial B,
\end{aligned}
$$

where $F \in C\left(\mathbb{R}^{+}, \mathbb{R}^{+}\right)$.

It is clear that the radial solutions of (5) are equivalent to the solutions of the following problem:

$$
\begin{aligned}
\left(\left(u^{\prime}\right)^{N}\right)^{\prime} & =\gamma N r^{N-1} h(r) F(-u), \quad r \in(0,1), \\
u^{\prime}(0) & =u(1)=0,
\end{aligned}
$$

where $\gamma$ is a positive parameter and $h$ and $F=f_{1}+f_{2}$ with $f_{1}, f_{2} \in C\left(\mathbb{R}^{+}, \mathbb{R}^{+}\right)$satisfy the following.

(A0) $h(x) \in C(\bar{B})$ is radially symmetric and $h(r) \geq 0$, $h(r) \neq \equiv \quad 0$ on any subinterval of $[0,1]$, where $r=|x|$ with $x \in \bar{B}$.
(A1) $f_{1} \in C\left(\mathbb{R}^{+}, \mathbb{R}^{+}\right)$and there exist $a_{0}, a^{0}, a_{\infty}, a^{\infty} \in$ $(0, \infty)$ such that

$$
\begin{gathered}
a_{0} \leq \liminf _{s \rightarrow 0^{+}} \frac{f_{1}(s)}{s^{N}}<\limsup _{s \rightarrow 0^{+}} \frac{f_{1}(s)}{s^{N}} \leq a^{0}, \\
a_{\infty} \leq \liminf _{s \rightarrow+\infty} \frac{f_{1}(s)}{s^{N}}<\limsup _{s \rightarrow+\infty} \frac{f_{1}(s)}{s^{N}} \leq a^{\infty} .
\end{gathered}
$$

(A2) $f_{2} \in C\left(\mathbb{R}^{+}, \mathbb{R}^{+}\right)$and $f_{2}(s)>0$ for $s \in(0, \infty)$ and there exist $f_{2}^{0}, f_{2}^{\infty} \in(0, \infty)$ such that

$$
\begin{aligned}
f_{2}^{0} & =\lim _{s \rightarrow 0^{+}} \frac{f_{2}(s)}{s^{N}}, \\
f_{2}^{\infty} & =\lim _{s \rightarrow+\infty} \frac{f_{2}(s)}{s^{N}} .
\end{aligned}
$$

The rest of this paper is arranged as follows. In Section 2, we establish the bifurcation results which bifurcate from the trivial solutions axis and from infinity for problem (2), respectively. In Section 3, on the basis of the interval bifurcation result (Theorems 3 and 6), we give the intervals for the parameter $\gamma$ which ensure existence of single or multiple strictly convex solutions for problem (6) under the assumptions of (A1)-(A2).

\section{Global Interval Bifurcation}

Let $Y=C[0,1]$ with the norm $\|u\|_{\infty}=\max _{r \in[0,1]}|u(r)|$. Let $E:=\left\{u(r) \in C^{1}(0,1) \mid u^{\prime}(0)=u(1)=0\right\}$ with the usual norm $\|u\|=\max \left\{\|u\|_{\infty},\left\|u^{\prime}\right\|_{\infty}\right\}$. Let $P^{+}=\{u \in E: u(r)>0, r \in$ $(0,1)\}$. Set $K^{+}=\mathbb{R} \times P^{+}$under the product topology.

Now, we consider the following eigenvalue problem:

$$
\begin{aligned}
\left(\left(-v^{\prime}\right)^{N}\right)^{\prime} & =\lambda N r^{N-1} m(r) v^{N}, \quad \text { in } r \in(0,1), \\
u^{\prime}(0) & =u(1)=0 .
\end{aligned}
$$

By [16, (4.2) of Section 4, p.11], the same proof as in Theorem 1.1 of [14], we can show that problem (9) possesses the first eigenvalue $\lambda_{1}$ which is positive, simple, and unique, and the corresponding eigenfunctions are positive in $(0,1)$ and concave on $[0,1]$.

By Sections 3-4 in [16], with a simple transformation $v=$ $-u$, problem (2) can be equivalently written as

$$
\begin{array}{rl}
\left(\left(-v^{\prime}\right)^{N}\right)^{\prime} & =\lambda N r^{N-1} m(r) v^{N}+N r^{N-1} F\left(r, v, v^{\prime}, \lambda\right), \\
r & r(0,1), \\
v^{\prime}(0) & =v(1)=0,
\end{array}
$$

where $m$ satisfies (H0) when $F \in C\left([0,1] \times\left(\mathbb{R}^{+}\right)^{3}, \mathbb{R}^{+}\right)$satisfies (H2). According to Rabinowitz [21], using the same method to prove [16, Theorems 4.1 and 4.2] with obvious changes, we may get the following global bifurcation result.

Lemma 1 ([16, Theorem 4.2]). Assume that (HO) holds and $F$ satisfies (H3). Then $\left(\lambda_{1}, 0\right)$ is the unique bifurcation point 
of problem (10) and there exists an unbounded bifurcation continuum $C \subseteq\left(K^{+} \cup\left\{\left(\lambda_{1}, 0\right)\right\}\right)$ of solutions to problem (10) emanating from $\left(\lambda_{1}, 0\right)$.

Hence, $F=m(r) f_{1}+f_{2}$, where $f_{1}, f_{2}$ satisfy conditions (H1) and (H3), respectively. Let $\mathcal{S}$ denote the closure in $\mathbb{R} \times E$ of the set of nontrivial solutions $(\lambda, v)$ of (10) with $v \in P^{+}$under the assumptions of (H0), (H1), and (H3). By an argument similar to that of [16, Lemma 4.1] with obvious changes, we can show that the following existence and uniqueness theorem is valid for problem (10).

Lemma 2 ([16, Lemma 4.1]). If $(\lambda, v)$ is a solution of $(10)$ under the assumptions of (HO), (H1), and (H3) and $v$ has a double zero, then $v \equiv 0$.

The first main result for (10) is the following theorem.

Theorem 3. Let (HO), (H1), and (H3) hold. Let $I_{1}^{0}=\left[\lambda_{1}-\right.$ $\left.a^{0}, \lambda_{1}-a_{0}\right]$. The component $\mathscr{C}$ of $\mathcal{S} \cup\left(I_{1}^{0} \times\{0\}\right)$ contains $I_{1}^{0} \times\{0\}$, such that

(i) $\mathscr{C} \subset\left(K^{+} \cup\left(I_{1}^{0} \times\{0\}\right)\right)$;

(ii) $\mathscr{C}$ is unbounded.

To prove Theorem 3, we introduce the following auxiliary approximate problem:

$$
\begin{aligned}
\left(\left(-v^{\prime}\right)^{N}\right)^{\prime}= & \lambda N r^{N-1} m(r) v^{N} \\
+ & N r^{N-1} m(r) f_{1}\left(r, v|v|^{\epsilon}, v^{\prime}, \lambda\right) \\
& +N r^{N-1} f_{2}\left(r, v, v^{\prime}, \lambda\right), \quad r \in(0,1), \\
v^{\prime}(0)= & v(1)=0 .
\end{aligned}
$$

To prove Theorem 3, the next lemma will play a key role.

Lemma 4. Let $\epsilon_{n}, 0<\epsilon_{n}<1$, be a sequence converging to 0 . If there exists a sequence $\left(\lambda_{n}, v_{n}\right) \in K^{+}$such that $\left(\lambda_{n}, v_{n}\right)$ is a nontrivial solution of problem (11) corresponding to $\epsilon=\epsilon_{n}$ and $\left(\lambda_{n}, v_{n}\right)$ converges to $(\lambda, 0)$ in $\mathbb{R} \times E$, then $\lambda \in I_{1}^{0}$.

Proof. Let $w_{n}=v_{n} /\left\|v_{n}\right\|$; then, $w_{n}$ satisfies the problem

$$
\begin{aligned}
\left(\left(-w_{n}^{\prime}\right)^{N}\right)^{\prime}= & \lambda N r^{N-1} m(r) w_{n}^{N}+N r^{N-1} m(r) f_{1}^{n}(r) \\
& +N r^{N-1} f_{2}^{n}(r), \quad r \in(0,1) \\
w_{n}^{\prime}(0)= & w_{n}(1)=0, \\
f_{1}^{n}(r)= & \frac{f_{1}\left(r, v_{n}(r)\left|v_{n}(r)\right|^{\epsilon}, v_{n}^{\prime}(r), \lambda\right)}{\left\|v_{n}\right\|^{N}} \\
f_{2}^{n}(r)= & \frac{f_{2}\left(r, v_{n}(r), v_{n}^{\prime}(r), \lambda\right)}{\left\|v_{n}\right\|^{N}}
\end{aligned}
$$

Let $\overline{f_{2}}(r, v, \lambda)=\max _{0 \leq|s| \leq v}\left|f_{2}(r, s, \lambda)\right|$ for all $r \in(0,1)$ and $\lambda$ on bounded sets, and then $\overline{f_{2}}$ is nondecreasing with respect to $v$ and $\lim _{v \rightarrow 0^{+}}\left(\overline{f_{2}}(r, v, \lambda) / v^{N}\right)=0$ uniformly for $r \in[0,1]$ and $\lambda$ on bounded sets. By an argument similar to that of $[16$, (4.7)] with obvious changes, we can show that $f_{2}^{n}(r) \rightarrow 0$ as $\|v\| \rightarrow 0$ uniformly for $r \in(0,1)$ and $\lambda$ on bounded sets. By (H1), we have that $w_{n}$ is convergent in $E$. Without loss of generality, we may assume that $w_{n} \rightarrow w$ in $E$ with $\|w\|=1$. Obviously, we have $w \in P^{+}$.

Now, we deduce the boundedness of $\lambda$. Let $\psi \in P^{+}$be an eigenfunction of problem (9) with $F=0$ corresponding to $\lambda_{1}$.

Similar to (4.12) in Lemma 4.5 of [16], by some simple calculations, we have that

$$
\int_{0}^{1}\left(\frac{w_{n}^{N+1}\left(-\psi^{\prime}\right)^{N}}{\psi^{N}}-w_{n}\left(-w_{n}^{\prime}\right)^{N}\right)=A+B
$$

where

$$
\begin{aligned}
A= & \int_{0}^{1}\left[\left(\lambda_{1}-\lambda_{n}\right) m(r)-\frac{f_{1}^{n}(r)}{w_{n}^{N}}-\frac{f_{2}^{n}(r)}{w_{n}^{N}}\right] \\
& \cdot r^{N-1} N w_{n}^{N+1} d r \\
B= & {\left[\left(-w_{n}^{\prime}\right)^{N+1}+N\left(\frac{-w_{n} \psi^{\prime}}{\psi}\right)^{N+1}+(N+1)\right.} \\
& \left.\cdot w_{n}^{N} w_{n}^{\prime}\left(\frac{-\psi^{\prime}}{\psi}\right)^{N}\right] .
\end{aligned}
$$

Similar to the proof of (4.12) in Lemma 4.5 of [16], we can show that the left-hand side of (14) equals 0. The Young's inequality implies that $B \geq 0$. It follows that

$$
\begin{aligned}
& \int_{0}^{1}\left[\left(\lambda_{1}-\lambda_{n}\right) m(r)-\frac{f_{1}^{n}(r)}{w_{n}^{N}}-\frac{f_{2}^{n}(r)}{w_{n}^{N}}\right] \\
& \cdot r^{N-1} N w_{n}^{N+1} d r \leq 0 .
\end{aligned}
$$

Similarly, we can also show that

$$
\begin{aligned}
& \int_{0}^{1}\left[\left(\lambda_{n}-\lambda_{1}\right) m(r)+\frac{f_{1}^{n}(r)}{w_{n}^{N}}+\frac{f_{2}^{n}(r)}{w_{n}^{N}}\right] \\
& \cdot r^{N-1} N \psi^{N+1} d r \leq 0 .
\end{aligned}
$$

Similar to [17, Lemma 1], we can easily show that

$$
\begin{aligned}
& a_{0} \int_{0}^{1} m N r^{N-1} w^{N+1} d r \leq \int_{0}^{1} \frac{f_{1}^{n}(r)}{w_{n}^{N}} m N r^{N-1} w_{n}^{N+1} d r \\
& \quad \leq a^{0} \int_{0}^{1} m N r^{N-1} w^{N+1} d r
\end{aligned}
$$

for $n$ large enough.

It follows from (16) and (18) that

$$
\int_{0}^{1}\left(\lambda_{1}-\lambda-a^{0}\right) m(r) N r^{N-1} w^{N+1} \leq 0,
$$

which implies $\lambda \geq \lambda_{1}-a^{0}$.

Similarly, it follows from (17) and (18) that $\lambda \leq \lambda_{1}-a_{0}$.

Therefore, we have that $\lambda \in I_{1}^{0}$. 
Proof of Theorem 3. We divide the rest of the proofs into two steps.

Step 1. Using a similar method to prove [20, Theorem 1.1] with obvious changes, we may prove that $\mathscr{C} \subset\left(K^{+} \cup\left(I_{1}^{0} \times\right.\right.$ $\{0\}))$.

Step 2. We prove that $\mathscr{C}$ is unbounded. Suppose on the contrary that $\mathscr{C}$ is bounded. Using a similar method to prove [20, Theorem 1.1] with obvious changes, we can find a neighborhood $\mathcal{O}$ of $\mathscr{C}$ such that $\partial \mathscr{O} \cap \mathcal{S}=\emptyset$.

In order to complete the proof of this theorem, we consider problem (11). For $\epsilon>0$, it is easy to show that the nonlinear term $f\left(r, v|v|^{\epsilon}, \lambda\right)+g(r, v, \lambda)$ satisfies condition (H3). Let

$$
\mathcal{S}_{\epsilon}=\overline{\{(\lambda, v):(\lambda, v) \text { satisfies }(11), v \not \equiv 0}^{\mathbb{R} \times E} .
$$

By Lemma 1 , there exists an unbounded continuum $\mathscr{C}_{\epsilon}$ of $\delta_{\epsilon}$ bifurcating from $\left(\lambda_{1}, 0\right)$ such that

$$
\mathscr{C}_{\epsilon} \subset\left(K^{+} \cup\left\{\left(\lambda_{1}, 0\right)\right\}\right) .
$$

So there exists $\left(\lambda_{\epsilon}, v_{\epsilon}\right) \in \mathscr{C}_{\epsilon} \cap \partial \mathcal{O}$ for all $\epsilon>0$. Since $\mathcal{O}$ is bounded in $\mathbb{R} \times P^{+}$, (11) shows that $\left(\lambda_{\epsilon}, v_{\epsilon}\right)$ is bounded in $\mathbb{R} \times C^{2}$ independently of $\epsilon$. By the compactness of $G_{N}$, one can find a sequence $\epsilon_{n} \rightarrow 0$ such that $\left(\lambda_{\epsilon_{n}}, v_{\epsilon_{n}}\right)$ converges to a solution $(\lambda, v)$ of (11). So, $v \in \overline{P^{+}}$. If $v \in \partial P^{+}$, then from Lemma 2 it follows that $v \equiv 0$. By Lemma $4, \lambda \in I_{1}^{0}$, which contradicts the definition of $\mathcal{O}$. On the other hand, if $v \in P^{+}$, then $(\lambda, v) \in \mathcal{S} \cap \partial \mathcal{O}$ which contradicts $\mathcal{S} \cap \partial \mathcal{O}=\emptyset$.

From Theorem 3 and its proof, we can easily get a corollary.

Corollary 5. Let (HO), (H1), and (H3) hold. There exists a subcontinuum $\mathscr{D}$ of solutions of (10) in $\mathbb{R} \times E$, bifurcating from $I_{1}^{0} \times\{0\}$, such that

(i) $\mathscr{D} \subset\left(K^{+} \cup\left(I_{1}^{0} \times\{0\}\right)\right)$;

(ii) $\mathscr{D}$ is unbounded.

We add the points $\{(\lambda, \infty) \mid \lambda \in \mathbb{R}\}$ to space $\mathbb{R} \times$ E. Let $\mathscr{T}$ denote the closure in $\mathbb{R} \times E$ of the set of nontrivial solutions $(v, \lambda)$ of (10) under conditions (H2) and (H4) with $v \in P^{+}$. Let $S_{N}$ denote the spectral set of problem (9). Let $\bar{I}_{\infty}=\left[\bar{\lambda}-a^{\infty}, \bar{\lambda}-a_{\infty}\right]$, where $\bar{\lambda} \in S_{N} \backslash\left\{\lambda_{1}\right\}$.

According to Rabinowitz [22], our second main result for (10) is the following theorem.

Theorem 6. Let (HO), (H2), and (H4) hold. Let $I_{1}^{\infty}=\left[\lambda_{1}-\right.$ $\left.a^{\infty}, \lambda_{1}-a_{\infty}\right]$. There exists a connected component $\mathscr{D}$ of $\mathscr{T} \cup$ $\left(I_{1}^{\infty} \times\{\infty\}\right)$, containing $I_{1}^{\infty} \times\{\infty\}$. Moreover, if $\Lambda \subset \mathbb{R}$ is an interval such that $\Lambda \cap\left(\bigcup_{\bar{\lambda}_{\in} S_{N} \backslash\left\{\lambda_{1}\right\}}\left(\bar{I}_{\infty} \cup I_{1}^{\infty}\right)\right)=I_{1}^{\infty}$ and $\mathscr{M}$ is a neighborhood of $I_{1}^{\infty} \times\{\infty\}$ whose projection on $\mathbb{R}$ lies in $\Lambda$ and whose projection on $E$ is bounded away from 0 , then either

$\left(1^{\circ}\right) \mathscr{D}-\mathscr{M}$ is bounded in $\mathbb{R} \times E$ in which case $\mathscr{D}-\mathscr{M}$ meets $\mathscr{R}=\{(\lambda, 0) \mid \lambda \in \mathbb{R}\}$;

$\left(2^{\circ}\right) \mathscr{D}-\mathscr{M}$ is unbounded.
If $\left(2^{\circ}\right)$ occurs and $\mathscr{D}-\mathscr{M}$ has a bounded projection on $\mathbb{R}$, then $\mathscr{D}-\mathscr{M}$ meets $\bar{I}_{\infty} \times\{\infty\}$. Moreover, there exists a neighborhood $\mathcal{N} \subset \mathscr{M}$ of $I_{1}^{\infty} \times\{\infty\}$ such that $(\mathscr{D} \cap \mathcal{N}) \subset$ $\left(K^{+} \cup\left(I_{1}^{\infty} \times\{\infty\}\right)\right)$.

Proof. We use a similar method to prove [18, Theorem 2.2] with obvious changes, but we give a rough sketch of the proof for readers' convenience. If $(\lambda, v) \in \mathscr{T}$ with $\|v\| \neq 0$, dividing (10) by $\|v\|^{2}$ and setting $w=v /\|v\|^{2}$ yield

$$
\begin{aligned}
\left(\left(-w^{\prime}\right)^{N}\right)^{\prime}= & \lambda N r^{N-1} m(r) w^{N} \\
& +\frac{N r^{N-1} F\left(r, v, v^{\prime}, \lambda\right)}{\|v\|^{2 N}}, \quad r \in(0,1), \\
w^{\prime}(0)= & w(1)=0 .
\end{aligned}
$$

Define

$$
\begin{aligned}
& \tilde{h}\left(r, w, w^{\prime}, \lambda\right) \\
& \quad= \begin{cases}\|w\|^{2 N} h\left(r, \frac{w}{\|w\|^{2}}, \frac{w^{\prime}}{\|w\|^{2}}, \lambda\right), & \text { if } w \neq 0, \\
0, & \text { if } w=0\end{cases}
\end{aligned}
$$

Clearly, (22) is equivalent to

$$
\begin{aligned}
&\left(\left(-w^{\prime}\right)^{N}\right)^{\prime}= \lambda N r^{N-1} m(r) w^{N} \\
&+N r^{N-1} m(r) \widetilde{f_{1}}\left(r, w, w^{\prime}, \lambda\right) \\
&+N r^{N-1} \widetilde{f}_{2}\left(r, w, w^{\prime}, \lambda\right), \quad r \in(0,1), \\
& w^{\prime}(0)=w(1)=0 .
\end{aligned}
$$

It is obvious that $(\lambda, 0)$ is always the solution of $(24)$. By simple computation, we can show that assumptions (H2) and (H4) imply that $\widetilde{f_{1}}, \widetilde{f_{2}}$ satisfy (H1) and (H3). Now, applying Theorem 3 to problem (24), we have that the component $\mathscr{C}$ of $\mathcal{S} \cup\left(I_{1}^{0} \times\{0\}\right)$, containing $I_{1}^{0} \times\{0\}$, is unbounded and lies in $K^{+} \cup\left(I_{1}^{0} \times\{0\}\right)$. Under the inversion $w \rightarrow w /\|w\|^{2}=v, \mathscr{C} \rightarrow \mathscr{D}$ satisfying problem (10). By an argument similar to that of [18, Theorem 2.3], we can prove the existence of a neighborhood $\mathscr{N} \subset \mathscr{M}$ of $I_{1}^{\infty} \times\{\infty\}$ such that $(\mathscr{D} \cap \mathcal{N}) \subset\left(K^{+} \cup\left(I_{1}^{\infty} \times\right.\right.$ $\{\infty\}))$.

\section{Applications}

In this section, we shall investigate the existence and multiplicity of convex solutions of problem (6). With a simple transformation $v=-u$, problem (6) can be written as

$$
\begin{aligned}
\left(\left(-v^{\prime}\right)^{N}\right)^{\prime} & =\gamma N r^{N-1} h(r) F(v), \quad r \in(0,1), \\
v^{\prime}(0) & =v(1)=0 .
\end{aligned}
$$

By [16], in order to prove our main results, we need the following Sturm type comparison result. 
Lemma 7 (see [16, Lemma 4.6]). Let $b_{i}(r) \in C(0,1), i=1,2$, such that $b_{2}(r) \geq b_{1}(r)$ for $r \in(0,1)$ and the inequality is strict on some subset of positive measure in $(0,1)$. Also, let $v_{1}$ and $v_{2}$ be solutions of the following differential equations:

$$
\begin{aligned}
\left(\left(-v^{\prime}\right)^{N}\right)^{\prime} & =b_{i}(r) v^{N}, \quad r \in(0,1), i=1,2, \\
v^{\prime}(0) & =v(1)=0,
\end{aligned}
$$

respectively. If $v_{1} \neq 0$ in $(0,1)$, then $v_{2}$ has at least one zero in $(0,1)$.

The main results of this section are the following theorems.

Theorem 8. Let (A0), (A1), and (A2) hold. If $f_{2}^{0}>-a_{0}$ and $f_{2}^{\infty}>-a_{\infty}$, for either

$$
\frac{\lambda_{1}}{f_{2}^{0}+a_{0}}<\gamma<\frac{\lambda_{1}}{f_{2}^{\infty}+a^{\infty}}
$$

or

$$
\frac{\lambda_{1}}{f_{2}^{\infty}+a_{\infty}}<\gamma<\frac{\lambda_{1}}{f_{2}^{0}+a^{0}},
$$

then problem (6) has at least one solution $u$ such that it is negative and strictly convex in $(0,1)$.

Theorem 9. Let (A0), (A1), and (A2) hold. If $f_{2}^{0}>-a_{0}$ and $-a^{\infty} \leq f_{2}^{\infty} \leq-a_{\infty}$, for

$$
\frac{\lambda_{1}}{f_{2}^{0}+a_{0}}<\gamma<\frac{\lambda_{1}}{f_{2}^{\infty}+a^{\infty}}
$$

then problem (6) has at least one solution $u$ such that it is negative and strictly convex in $(0,1)$.

Theorem 10. Let (A0), (A1), and (A2) hold. If $g_{0}>-a_{0}$ and $f_{2}^{\infty} \leq-a^{\infty}$, for

$$
\gamma>\frac{\lambda_{1}}{f_{2}^{0}+a_{0}},
$$

then problem (6) has at least one solution $u$ such that it is negative and strictly convex in $(0,1)$.

Theorem 11. Let (A0), (A1), and (A2) hold. If $-a^{0}<f_{2}^{0} \leq a_{0}$ and $f_{2}^{\infty}>-a_{\infty}$, for

$$
\frac{\lambda_{1}}{f_{2}^{\infty}+a_{\infty}}<\gamma<\frac{\lambda_{1}}{f_{2}^{0}+a^{0}},
$$

then problem (6) has at least one solution $u$ such that it is negative and strictly convex in $(0,1)$.

Theorem 12. Let (A0), (A1), and (A2) hold. If $-a^{0} \leq g_{0}$ and $f_{2}^{\infty}>-a_{\infty}$, for

$$
\gamma>\frac{\lambda_{1}}{f_{2}^{\infty}+a_{\infty}},
$$

then problem (6) has at least one solution $u$ such that it is negative and strictly convex in $(0,1)$.
Remark 13. Note that if $f \equiv 0$, then Theorem 8 is equivalent to Theorem 4.1 of [15] or Theorem 5.1 of [16]. In this sense, Theorem 8 is also a generalization of Theorem 4.1 of [15] or Theorem 5.1 of [16].

Proof of Theorem 8. It suffices to prove that problem (25) has at least one solution $v$ such that it is positive and strictly concave in $(0,1)$.

Firstly, we study the bifurcation phenomena for the following eigenvalue problem:

$$
\begin{aligned}
\left(\left(-v^{\prime}\right)^{N}\right)^{\prime}= & \lambda \gamma N r^{N-1} h(r) f_{2}(v) \\
& +\gamma N r^{N-1} h(r) f_{1}(v), \quad r \in(0,1), \\
v^{\prime}(0)= & v(1)=0,
\end{aligned}
$$

where $\lambda>0$ is a parameter.

Let $\zeta \in C\left(\mathbb{R}^{+}, \mathbb{R}^{+}\right)$be such that

$$
f_{2}(s)=f_{2}^{0} s^{N}+\zeta(s)
$$

with $\lim _{s \rightarrow 0^{+}} \zeta(s) / s^{N}=0$. Let $\bar{\zeta}(v)=\max _{0 \leq|s| \leq v}|\zeta(s)|$; then, $\bar{\zeta}(v)$ is nondecreasing and

$$
\lim _{s \rightarrow 0^{+}} \frac{\bar{\zeta}(s)}{s^{N}}=0 .
$$

Further, it follows from (35) that

$$
\frac{|\zeta(v)|}{\|v\|^{N}} \leq \frac{\bar{\zeta}(|v|)}{\|v\|^{N}} \leq \frac{\bar{\zeta}\left(\|r\|_{\infty}\right)}{\|v\|^{N}} \leq \frac{\bar{\zeta}(\|r\|)}{\|v\|^{N}}
$$

as $\|v\| \longrightarrow 0$.

Hence, (33), (34), and (36) imply that conditions (H1) and (H3) hold. Moreover, we have that $I_{1}^{0}=\left[\lambda_{1} / \gamma f_{2}^{0}-\right.$ $\left.a^{0} / f_{2}^{0}, \lambda_{1} / \gamma f_{2}^{0}-a_{0} / f_{2}^{0}\right]$.

By Theorem 3, there is a distinct unbounded component $\mathscr{D}_{0}$ of $\mathcal{S} \cup\left(I_{1}^{0} \times\{0\}\right)$, containing $I_{1}^{0} \times\{0\}$ and lying in $\left(K^{+} \cup\right.$ $\left.\left(I_{1}^{0} \times\{0\}\right)\right)$.

Let $\xi \in C\left(\mathbb{R}^{+}, \mathbb{R}^{+}\right)$be such that

$$
f_{2}(s)=f_{2}^{\infty} s^{N}+\xi(s)
$$

with $\lim _{s \rightarrow+\infty} \xi(s) / s^{N}=0$. Let $\bar{v}=\max _{0 \leq|s| \leq v}|\xi(s)|$, and then $\bar{\xi}$ is nondecreasing.

Define

$$
\widetilde{v}=\max _{v / 2 \leq|s| \leq v}|\xi(s)| .
$$

Then, we can see that

$$
\begin{aligned}
\lim _{s \rightarrow+\infty} \frac{\tilde{\xi}(s)}{s^{N}} & =0, \\
\bar{v} & \leq \frac{\bar{v}}{2}+\widetilde{v} .
\end{aligned}
$$


It is not difficult to verify that $\bar{v} / s$ is bounded in $\mathbb{R}^{+}$. From this fact and (37), it follows that

$$
\limsup _{s \rightarrow+\infty} \frac{\bar{\xi}(v)}{v^{N}} \leq \limsup _{s \rightarrow+\infty} \frac{\bar{\xi}(v / 2)}{v^{N}}=\limsup _{s \rightarrow+\infty} \frac{\bar{\xi}(v)}{v^{N}},
$$

where $t=u / 2$. So, we have

$$
\lim _{s \rightarrow+\infty} \frac{\bar{\xi}(s)}{s^{N}}=0 .
$$

Further, it follows from (41) that

$$
\frac{|\xi(v)|}{\|v\|^{N}} \leq \frac{\bar{\xi}(|v|)}{\|v\|^{N}} \leq \frac{\bar{\xi}\left(\|v\|_{\infty}\right)}{\|v\|^{N}} \leq \frac{\bar{\xi}(\|v\|)}{\|v\|^{N}}
$$

as $\|v\| \longrightarrow+\infty$.

Hence, (33), (37), and (42) imply that conditions (H2) and (H4) hold. Moreover, we have that $I_{1}^{\infty}=\left[\lambda_{1} / \gamma f_{2}^{\infty}-\right.$ $\left.a^{\infty} / f_{2}^{\infty}, \lambda_{1} / \gamma f_{2}^{\infty}-a_{\infty} / f_{2}^{\infty}\right]$.

By Theorem 6, there is a distinct unbounded component $\mathscr{D}_{\infty}$ of $\mathscr{T} \cup\left(I_{1}^{\infty} \times\{\infty\}\right)$, which satisfies the alternates of Theorem 6. Moreover, there exists a neighborhood $\mathcal{N} \subset \mathscr{M}$ of $I_{1}^{\infty} \times\{\infty\}$ such that $\left(\mathscr{D}_{\infty} \cap \mathcal{N}\right) \subset\left(K^{+} \cup\left(I_{1}^{\infty} \times\{\infty\}\right)\right)$.

We claim that $\mathscr{D}_{0}=\mathscr{D}_{\infty}$. It suffices to show that $\mathscr{D}_{\infty}$ meets some point $\left(\lambda_{*}, 0\right)$ of $\mathscr{R}$. In fact, if this occurs, we can show that $\lambda_{*} \in I_{1}^{0}$. Suppose on the contrary that $\lambda_{*} \notin I_{1}^{0}$; hence, $\lambda_{*} \in \bar{I}_{0}$. So, $\left(\mathscr{D}_{\infty} \cap \mathcal{N}\right) \subset \mathscr{D}_{\infty} \subset \overline{\mathscr{D}}_{0} \subset\left((\mathbb{R} \times \bar{P}) \cup\left(\bar{I}_{0} \times\right.\right.$ $\{0\}))$, noting $\left(\mathscr{D}_{\infty} \cap \mathscr{N}\right) \cap(\mathbb{R} \times\{0\})=\emptyset$, which contradicts $\left(\mathscr{D}_{\infty} \cap \mathcal{N}\right) \subset\left(K^{+} \cup\left(I_{\infty} \times\{\infty\}\right)\right)$, where $\overline{\mathscr{T}}_{0}$ denotes the closure in $\mathbb{R} \times E$ of the set of nontrivial solutions $(\lambda, v)$ of $(25)$ under conditions (H1) and (H3) with $v \in \bar{P}$, where $\bar{P}=\{v \mid(\bar{\lambda}, v) \epsilon$ $\left.\left(S_{N} \backslash\left\{\lambda_{1}\right\}\right) \times E\right\}, \bar{I}_{0}=\left[\bar{\lambda} / \gamma f_{2}^{0}-a^{0} / f_{2}^{0}, \bar{\lambda} / \gamma f_{2}^{0}-a_{0} / f_{2}^{0}\right]$, where $\bar{\lambda} \in S_{N} \backslash\left\{\lambda_{1}\right\} . \overline{\mathscr{D}}_{0}$ is a connected component of $\overline{\mathscr{T}}_{0} \cup\left(\bar{I}_{0} \times\{0\}\right)$, containing $\bar{I}_{0} \times\{0\}$.

Hence, $\lambda_{*} \in I_{1}^{0}$, and it follows that $\mathscr{D}_{0}=\mathscr{D}_{\infty}$.

Next, we shall show that $\left(2^{\circ}\right)$ of Theorem 6 does not occur. Suppose on the contrary that $\left(2^{\circ}\right)$ of Theorem 6 occurs; then, we shall deduce a contradiction. Firstly, we show that $\mathscr{D}_{\infty}-\mathscr{M}$ has a bounded projection on $\mathbb{R}$. We may claim $\mathscr{D}_{\infty} \subset K^{+}$. If $\left(\mathscr{D}_{\infty}-\left(\mathscr{D}_{\infty} \cap \mathscr{N}\right)\right) \not \subset K^{+}$, then there exists $(\mu, v) \in\left(\mathscr{D}_{\infty}-\right.$ $\left.\left(\mathscr{D}_{\infty} \cap \mathcal{N}\right)\right) \cap\left(\mathbb{R} \times \partial P^{+}\right)$. Since $v \in \partial P^{+}$, by Lemma $4, v \equiv 0$; that is, $\left(1^{\circ}\right)$ of Theorem 6 occurs, which is a contradiction.

On the contrary, we suppose that $\left(\mu_{n}, v_{n}\right) \in \mathscr{D}_{\infty}-\mathscr{M}$ such that

$$
\lim _{n \rightarrow \infty} \mu_{n}=\infty
$$

It follows that

$$
\begin{aligned}
\left(\left(-v_{n}^{\prime}\right)^{N}\right)^{\prime}= & \mu_{n}^{N} \gamma N r^{N-1} h(r) f_{2}\left(v_{n}\right) \\
& +\gamma N r^{N-1} h(r) f_{1}\left(v_{n}\right), \quad r \in(0,1), \\
v_{n}^{\prime}(0)= & v_{n}(1)=0 .
\end{aligned}
$$

In view of (A1) and (A2), we have that $\lim _{n \rightarrow \infty}\left(\mu_{n}^{N} N r^{N-1} a(r)\left(f_{2}\left(v_{n}\right) / v_{n}^{N}\right)+N r^{N-1} a(r)\left(f_{1}\left(v_{n}\right) /\right.\right.$ $\left.\left.v_{n}^{N}\right)\right)=\infty$ for any $r \in(0,1)$. By the Sturm comparison
(Lemma 7), we get that $v_{n}$ has at least one zero in $(0,1)$ for $n$ large enough, and this contradicts the fact that $v_{n}$ does not change its sign in $(0,1)$.

In the following, we show that the case of $\mathscr{D}_{\infty}-\mathscr{M}$ meeting $\bar{I}_{\infty} \times\{\infty\}$ is impossible. Assume on the contrary that $\mathscr{D}_{\infty}-\mathscr{M}$ meets $\bar{I}_{\infty} \times\{\infty\}$. So, there exists a neighborhood $\widetilde{\mathcal{N}} \subset \widetilde{\mathscr{M}}$ of $\bar{I}_{\infty}$ such that $\left(\mathscr{D}_{\infty}-\mathscr{M}\right) \cap\left(\widetilde{\mathcal{N}} \backslash\left(\bar{I}_{\infty} \times\{\infty\}\right)\right) \subset$ $(\mathbb{R} \times \bar{P})$, where $\widetilde{\mathscr{M}}$ is a neighborhood of $\bar{I}_{\infty} \times\{\infty\}$ which satisfies the assumptions of Theorem 6 , which contradicts $\mathscr{D}_{\infty} \subset K^{+}$, where $\bar{P}=\left\{v \mid(\bar{\lambda}, v) \in\left(S_{N} \backslash\left\{\lambda_{1}\right\}\right) \times E\right\}$ and $\bar{I}_{\infty}=$ $\left[\bar{\lambda} / \gamma f_{2}^{\infty}-a^{\infty} / f_{2}^{\infty}, \bar{\lambda} / \gamma f_{2}^{\infty}-a_{\infty} / f_{2}^{\infty}\right]$, where $\bar{\lambda} \in S_{N} \backslash\left\{\lambda_{1}\right\}$.

For simplicity, we write $\mathscr{D}=\mathscr{D}_{0}=\mathscr{D}_{\infty}$. It is clear that any solution of (33) of the form $(1, v)$ yields a solution $v$ of $(25)$.

By (27), we obtain

$$
\frac{\lambda_{1}^{N}}{\gamma f_{2}^{0}}-\frac{a_{0}}{f_{2}^{0}}<1<\frac{\lambda_{1}^{N}}{\gamma f_{2}^{\infty}}-\frac{a^{\infty}}{f_{2}^{\infty}}
$$

By (28), we have

$$
\frac{\lambda_{1}}{\gamma f_{2}^{\infty}}-\frac{a_{\infty}}{f_{2}^{\infty}}<1<\frac{\lambda_{1}}{\gamma f_{2}^{0}}-\frac{a^{0}}{f_{2}^{0}} .
$$

From $I_{1}^{0}=\left[\lambda_{1} / \gamma f_{2}^{0}-a^{0} / f_{2}^{0}, \lambda_{1} / \gamma f_{2}^{0}-a_{0} / f_{2}^{0}\right]$ and $I_{1}^{\infty}=$ $\left[\lambda_{1} / \gamma f_{2}^{\infty}-a^{\infty} / f_{2}^{\infty}, \lambda_{1} / \gamma f_{2}^{\infty}-a_{\infty} / f_{2}^{\infty}\right]$, it follows that the subsets $I_{1}^{0} \times E$ and $I_{1}^{\infty} \times E$ of $\mathbb{R} \times E$ can be separated by the hyperplane $\{1\} \times E$. Furthermore, we have $\mathscr{D}$ crossing the hyperplane $\{1\} \times E$ in $\mathbb{R} \times E$.

Proof of Theorems 9 and 10. The proof is similar to that of Theorem 8. In this case, it follows that (45) holds and (46) is impossible.

Proof of Theorems 11 and 12. The proof is similar to that of Theorem 8. In this case, it follows that (46) holds and (45) is impossible.

Remark 14. Note that if $-a^{0}<f_{2}^{0} \leq-a_{0}$ and $f_{2}^{\infty} \leq-a_{\infty}$ or $f_{2}^{0} \leq-a^{0}$ and $f_{2}^{\infty} \leq-a_{\infty},(45)$ and (46) are impossible, and it follows that the subsets $I_{1}^{0} \times E$ and $I_{1}^{\infty} \times E$ of $\mathbb{R} \times E$ cannot be separated by the hyperplane $\{1\} \times E$. In this case, we cannot give a suitable interval of $\gamma$ in which there exist nodal solutions for (25). It would be interesting to have more information about this case.

\section{Conflicts of Interest}

The author declares that there are no conflicts of interest regarding the publication of this paper.

\section{Acknowledgments}

The author was supported by the NSFC (no. 11561038) and Gansu Provincial National Science Foundation of China (no. 145RJZA087).

\section{References}

[1] D. Gilbarg and N. Trudinger, Elliptic Partial Differential Equations of Second Order, Springer, Verlag, Berlin, Heidelberg, 3rd edition, 2001. 
[2] X. J. Wang, "A class of fully nonlinear elliptic equations and related functionals," Indiana University Mathematics Journal, vol. 43, no. 1, pp. 25-54, 1994.

[3] K. Tso, "On a real Monge-Ampère functional," Inventiones Mathematicae, vol. 101, no. 2, pp. 425-448, 1990.

[4] A. V. Pogorelov, "The Diriehlet problem for the n-dimensional analogue of the Monge-Ampère equation," Soviet Mathematics - Doklady, vol. 12, pp. 1727-1731, 1971.

[5] S. Y. Cheng and S. T. Yao, "On the regularity of the MongeAmpère equation det $(\partial 2 u / \partial x i \partial x j)=\mathrm{F}(x, u)$," Communications on Pure and Applied Mathematics, vol. 3, pp. 41-68, 1977.

[6] P. L. Lions, "Sur les equations de Monge-Ampere. I," Manuscripta Mathematica, vol. 41, pp. 1-43, 1983.

[7] S. Y. Cheng and S.-T. Yau, "The real Monge-Ampère equation and affine flat structures," in Proceedings of the 1980 Beijing Symp. on Differential Geometry and Differential Equations, S. S. Cheng and W. T. Wu, Eds., pp. 339-370, Science Press, Beijing, China.

[8] P. Guan, N. S. Trudinger, and X. J. Wang, "On the Dirichlet problem for degenerate Monge-Ampere equations," Acta Mathematica, vol. 182, pp. 87-104, 1999.

[9] N. D. Kutev, "Nontrivial solutions for the equations of MongeAmpère type," Journal of Mathematical Analysis and Applications, vol. 132, no. 2, pp. 424-433, 1988.

[10] P. Delano, "Radially symmetric boundary value problems for real and complex elliptic Monge-Ampère equations," Journal of Differential Equations, vol. 58, pp. 318-344, 1985.

[11] L. Caffarelli, L. Nirenberg, and J. Spruck, “The Dirichlet problem for nonlinear second-order elliptic equations. I. MongeAmpère equation," Communications on Pure and Applied Mathematics, vol. 37, no. 3, pp. 369-402, 1984.

[12] S. Hu and H. Wang, "Convex solutions of boundary value problems arising from Monge-Ampère equations," Communications on Pure and Applied Mathematics, vol. 16, no. 3, pp. 705-720, 2006.

[13] H. Wang, "Convex solutions of boundary value problems," Journal of Mathematical Analysis and Applications, vol. 318, no. 1, pp. 246-252, 2006.

[14] P. L. Lions, "Two remarks on Monge-Ampère equations," Annali di Matematica Pura ed Applicata, vol. 142, no. 4, pp. 263-275, 1985.

[15] G. Dai and R. Ma, "Eigenvalue, bifurcation, convex solutions for Monge-Ampère equations," Topological Methods in Nonlinear Analysis, vol. 46, no. 1, pp. 135-163, 2015.

[16] G. Dai, "Two Whyburn type topological theorems and its applications to Monge-Ampère equations," Calculus of Variations and Partial Differential Equations, vol. 55, no. 4, pp. 1-28, 2016.

[17] H. Berestycki, "On some nonlinear Sturm-Liouville problems," Journal of Differential Equations, vol. 26, no. 3, pp. 375-390, 1977.

[18] R. Ma and G. Dai, "Global bifurcation and nodal solutions for a Sturm-Liouville problem with a nonsmooth nonlinearity," Journal of Functional Analysis, vol. 265, no. 8, pp. 1443-1459, 2013.

[19] G. Dai and R. Ma, "Bifurcation from intervals for SturmLiouville problems and its applications," Electronic Journal of Differential Equations, vol. 2014, no. 3, pp. 1-10, 2014.

[20] G. Dai and R. Ma, "Unilateral global bifurcation for p-Laplacian with non-p-1-linearization nonlinearity," Discrete and Continuous Dynamical Systems- Series A, vol. 35, no. 1, pp. 99-116, 2015.

[21] P. H. Rabinowitz, "Some global results for nonlinear eigenvalue problems," Journal of Functional Analysis, vol. 7, no. 3, pp. 487$513,1971$.
[22] P. H. Rabinowitz, "On bifurcation from infinity", Journal of Differential Equations, vol. 14, pp. 462-475, 1973. 


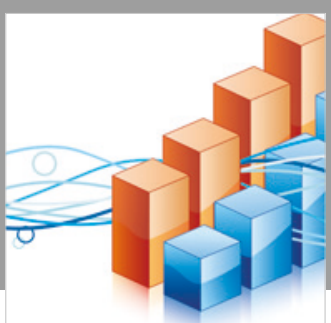

Advances in

Operations Research

\section{-n-m}
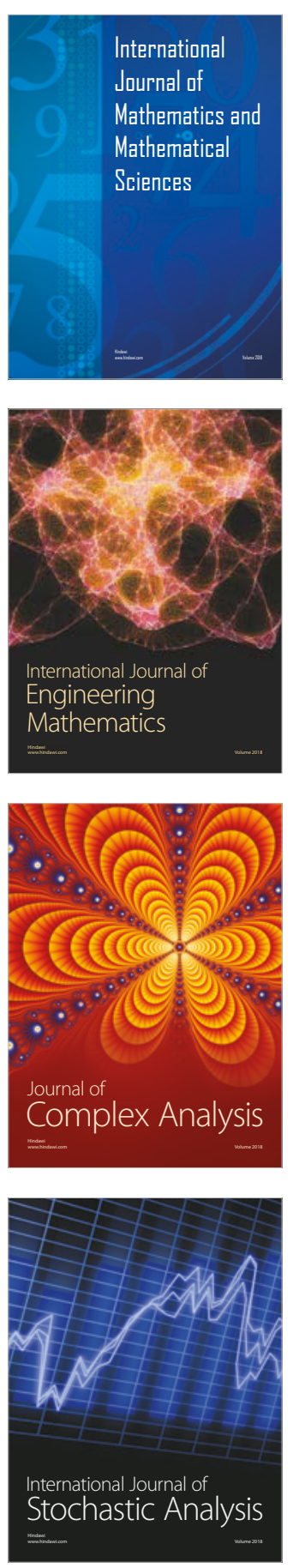
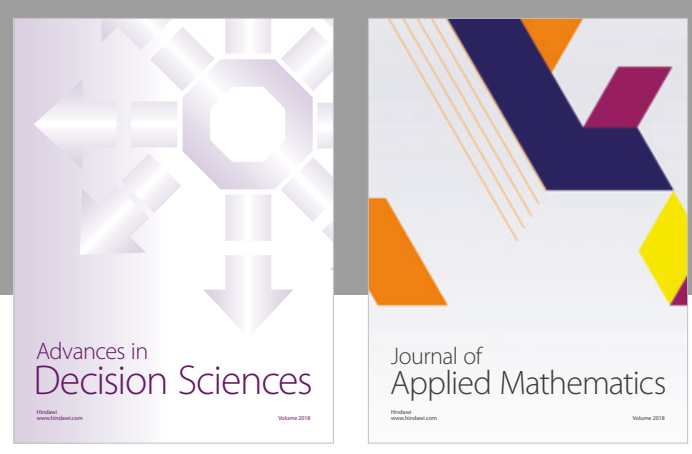

Journal of

Applied Mathematics
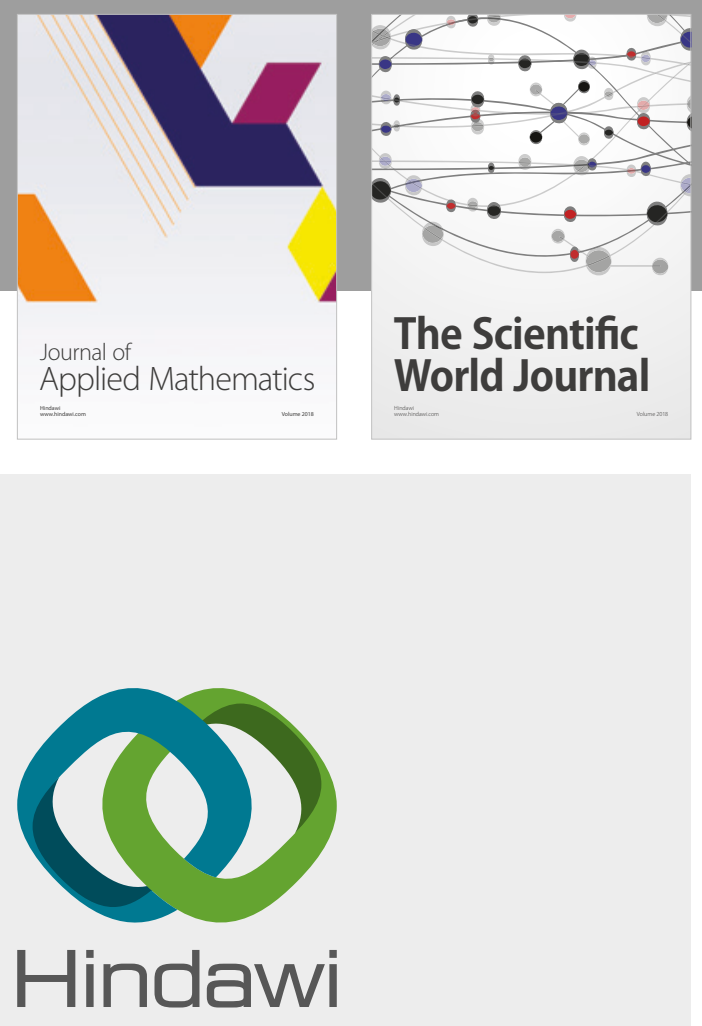

Submit your manuscripts at

www.hindawi.com

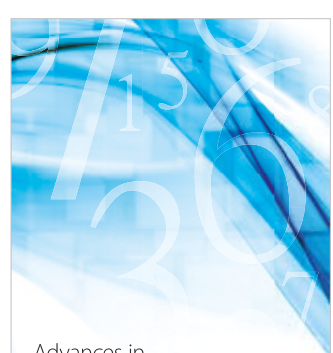

Advances in
Numerical Analysis
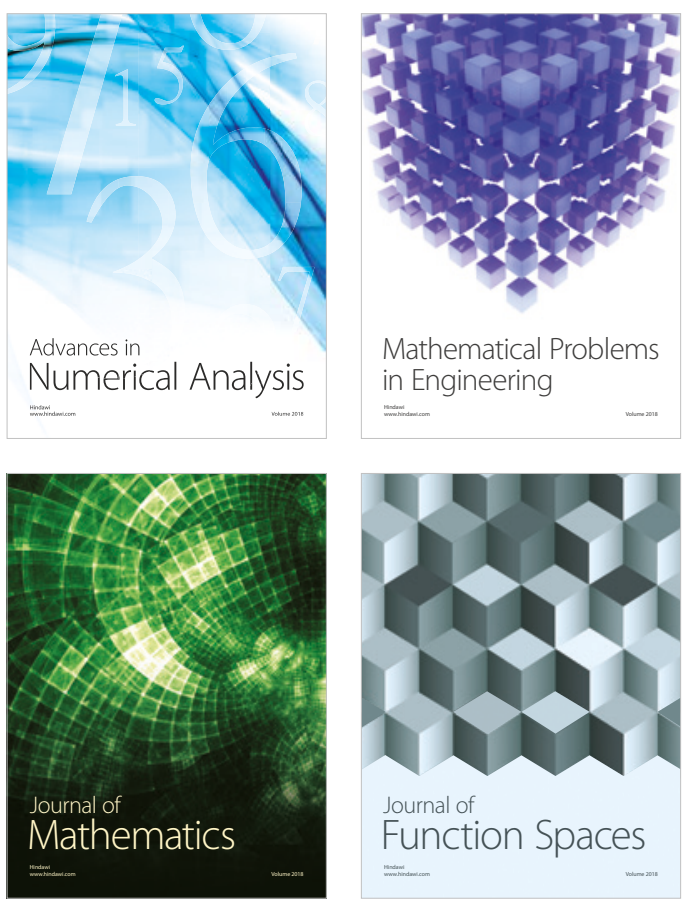

Mathematical Problems in Engineering

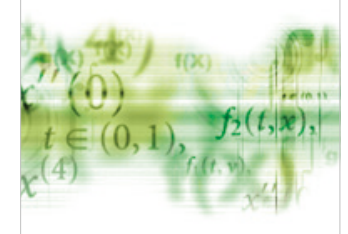

International Journal of

Differential Equations

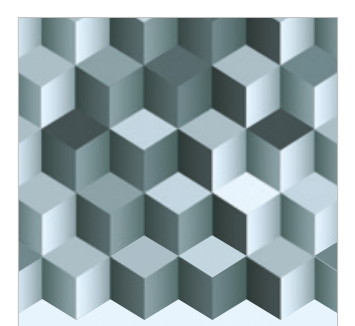

Journal of

Function Spaces

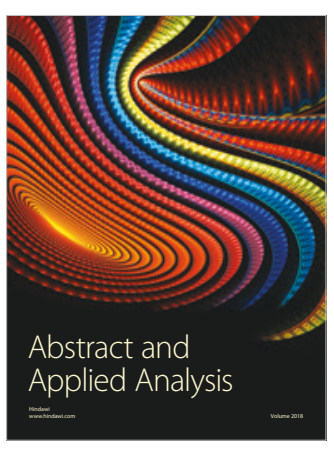

The Scientific

World Journal

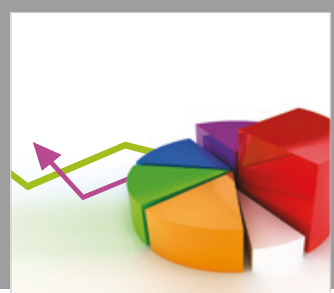

Journal of

Probability and Statistics
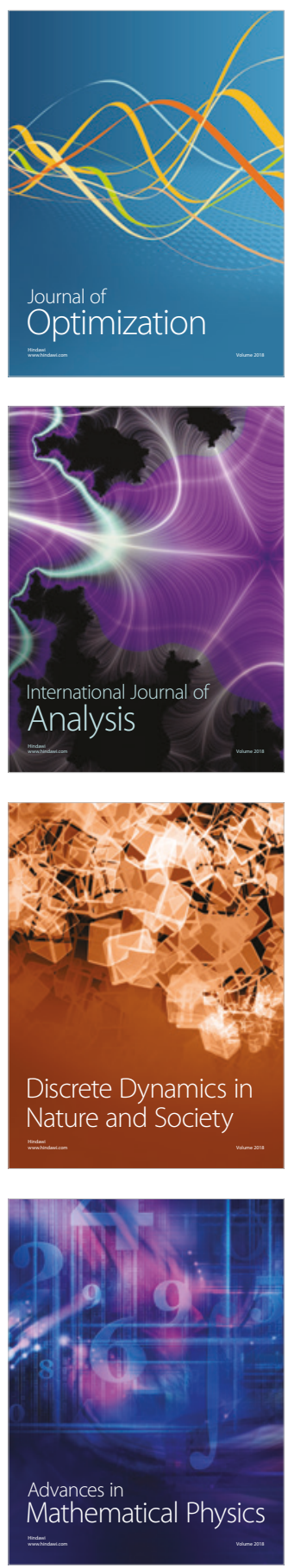